\title{
Dynamic State Estimation for Power System Control and Protection
}

\author{
IEEE Task Force on Power System Dynamic State and Parameter Estimation \\ Yu Liu, Member, IEEE, Abhinav Kumar Singh, Member, IEEE, Junbo Zhao (Chair), Senior Member, IEEE, \\ A. P. Meliopoulos, Fellow, IEEE, Bikash Pal, Fellow, IEEE, M. A. M. Ariff, Member, IEEE, Thierry Van Cutsem, \\ Fellow, IEEE, Mevludin Glavic, Senior Member, IEEE, Zhenyu Huang, Fellow, IEEE, Innocent Kamwa, Fellow, \\ IEEE, Lamine Mili, Life Fellow, IEEE, Abdul Saleem Mir, Member, IEEE, Ahmad Taha, Member, IEEE, \\ Vladimir Terzija, Fellow, IEEE, Shenglong Yu, Member, IEEE
}

\begin{abstract}
Dynamic state estimation (DSE) accurately tracks the dynamics of a power system and provides the evolution of the system state in real-time. This paper focuses on the control and protection applications of DSE, comprehensively presenting different facets of control and protection challenges arising in modern power systems. It is demonstrated how these challenges are effectively addressed with DSE-enabled solutions. As precursors to these solutions, reformulation of DSE considering both synchrophasor and sampled value measurements and comprehensive comparisons of DSE and observers have been presented. The usefulness and necessity of DSE based solutions in ensuring system stability, reliable protection and security, and resilience by revamping of control and protection methods are shown through examples, practical applications, and suggestions for further development.
\end{abstract}

Index Terms-Dynamic state estimation, Kalman filter, parameter estimation, stability, control, protection, synchrophasor measurements, sampled value measurements, synchronous generation, converter-based resources.

\section{INTRODUCTION}

Today's power systems are witnessing a rapid transition in

This work was partially supported by the Department of Energy Advanced Grid Modeling Program. (Corresponding author: Junbo Zhao)

Y. Liu, ShanghaiTech University, China (e-mail: liuyu@shanghaitech.edu.cn).

A. K. Singh and S. Mir are with University of Southampton, U.K (e-mail: a.k.singh@,soton.ac.uk, abdulsaleemmir@gmail.com).

J. Zhao is with Department of Electrical and Computer Engineering, Mississippi State University, MS, 39762, USA (e-mail: junbo@ece.msstate.edu).

A. P. Sakis Meliopoulos is with the School of Electrical and Computer Engineering, Georgia Institute of Technology, Atlanta, GA 30339 USA (e-mail: sakis.m@gatech.edu).

B. Pal is with the Department of Electrical and Electronic Engineering, Imperial College London, London SW7 2BU, U.K. (e-mail: b.pal@imperial.ac.uk).

M. Glavic and T. Van Cutsem are with Department of Electrical Engineering and Computer Science, University of Liege, Belgium (e-mail: t.vancutsem@ulg.ac.be).

M. Ariff is with Electrical and Electronic Engineering, Universiti Tun Hussein Onn Malaysia (UTHM), Malaysia, (e-mail: aifaa@uthm.edu.my)

Z. Huang is with the Pacific Northwest National Laboratory, Richland, WA 99352 USA (e-mail: zhenyu.huang@pnnl.gov).

I. Kamwa is with Laval University (e-mail: innocent.kamwa.1@ulaval.ca).

L. Mili is with the Bradley Department of Electrical and Computer Engi-

neering, Virginia Tech, Falls Church, VA 22043 USA (e-mail: 1 mili@vt.edu).

A. Taha is with the Department of Electrical and Computer Engineering,

The University of Texas at San Antonio (e-mail: ahmad.taha@utsa.edu).

V. Terzija is with the Department of Electrical and Electronic Engineering,

The University of Manchester (e-mail: vladimir.terzija@manchester.ac.uk).

$\mathrm{S}$. Yu is with the School of Engineering, Deakin University, Melbourne VIC

3216, Australia (e-mail: $\underline{\text { s.yu@ieee.org). }}$ generation technology from coal and gas-based non-renewable generation to wind and solar energy based renewable generation. Energy storage and modern power electronic loads penetrate power systems rapidly as well. As more and more of such converter-based resources (CBRs) are connected to the network, two major challenges emerge: (a) system response is faster and, therefore, its control should have a commensurate response speed, and (b) legacy protection functions that rely on the characteristics of conventional power systems (high fault currents and fault characteristics associated with synchronous generation) can be inadequate. Solutions to these challenges can be sought in dynamic state estimation (DSE) applications [1]. Specifically, DSE provides real-time operating states of the system at fast rates [2], which in turn can be utilized to fulfill the requirements for modern power systems protection and control.

Traditional schemes for power system control and protection are primarily based on a deterministic system model with the majority of electricity coming from a few centralized and synchronous sources of generation [3-6]. Such a system model has gradually become out of context due to the distributed, stochastic, and intermittent nature of renewable energy sources. In addition, stability, control and protection challenges introduced by the increased renewable integration include: 1 ) reduced system inertia, and 2) extreme variation in the timescales of system dynamics - from a few milliseconds or lower in the case of CBRs to a few minutes or higher in the case of boiler and long-term dynamics of synchronous generation. To deal with these challenges, it is necessary to consider the control of each dynamic component in the system individually, and how different components influence each other's dynamics, and how they should be controlled together holistically. As DSE provides state estimates at the required timescales, it can serve as a versatile tool to holistically control system trajectory, ensuring rotor-angle stability, frequency stability, voltage stability, resonance stability and converter-driven stability [3].

With the increasing penetration of CBRs in power systems, traditional schemes for power system protection face the following challenges: 1) Many traditional protection schemes depend on abrupt changes of voltages/currents during faults, e.g. overcurrent/undervoltage relays to detect faults; however, these characteristics may not be valid in systems with high penetration of CBRs [4]; 2) Phasor domain quantities are usually utilized in traditional relays, which can cause misoperation of relays during complex and unusual system transients in CBRdominated power systems; 3) Traditional protection schemes usually require complex coordination among relays, e.g. time overcurrent relays and 3-step distance relays, resulting in risks of mis-coordination; 4) Hidden failures, such as failures of instrumentation channels, can lead to misoperation of protection 
relays [5]. DSE is a valuable tool to overcome the above limitations. First, DSE can accurately track complex dynamics and provide accurate estimates of the dynamic states of the system, enabling precise extraction of fault characteristics in both the phasor domain and time domain [1]. In addition, for a specific protection zone, DSE can formulate the protection logic by systematically checking the consistency between the measurement and the dynamic model of the protection zone without coordination among relays [8]. Finally, with redundant measurements, DSE is able to identify and reject bad data, and therefore prevent relays from misoperation during hidden failures.

With the development of advanced measurement devices and substation automation, high-quality synchrophasor measurements and synchronized sampled value (SV) measurements provide more information at higher rates and enable DSE-based advanced control and protection schemes. Towards this end, this paper summarizes the joint efforts of the Task Force on Power System Dynamic State and Parameter Estimation, with an emphasis on DSE for power system control and protection. It has the following new insights:

- The existing DSE literature mainly focuses on electromechanical dynamics of synchronous machines while this paper extends it to consider the electromagnetic dynamics from CBRs.

- The relationships and differences for observer and DSE using Kalman filters have been extensively compared and discussed to clarify their advantages and disadvantages.

- The roles of DSE for control and protections have been thoroughly discussed with the support of numerical results in Sections IV and V.

- Several research directions have been offered to pave the way for further development in Section VI.

\section{DSE Formulation: SAMPLED VALUE MEASUREMENTS VERSUS PMU MEASUREMENTS}

Traditionally, power systems are dominated by synchronous generators. For these power systems, electromechanical oscillations with periods of a few seconds are very important. Although the detailed synchronous machine dynamic models involve electromagnetic transients, they are too fast as compared to the electromechanical oscillations and thus are neglected in the traditional dynamic studies and stability assessment [6]. With the increasing penetration of CBRs, such as distributed energy resources (DERs) and FACTS devices, to cite a few, the system dynamic responses are heavily dependent on the fastresponse power electronic devices and their controls, and converter-induced dynamics and stability issues start to dominate the system [6][9][10]. In addition, power electronics devices cause waveform distortions and deviations from near sinusoidal waveforms. Note that the time-scale of the CBRs can range from a few microseconds to several milliseconds, due to switching operations of the power electronics. Conventional phasor representation or quasi sinusoidal approximation is usually used for the study of electromechanical oscillations accounting for the synchronous machine oscillations and converter control dynamics (the dynamic phasors [11] or average models [12] could simplify the design of protection and control strategies for CBR systems). However, conventional phasor representation may not be suitable for the study of electromagnetic phenomena which represent the fast dynamics of the system. Hence, the for- mulation, measurement requirements, and potential applications of DSE are revisited in this paper, and the requirements for each application are defined.

Irrespective of the different time scales of power system dynamics, they can be described by differential and algebraic equations (DAEs). Note that for models described through partial differential equations (PDEs), such as wave propagations in transmission lines, appropriate discretization methods need to be utilized to convert PDEs into (1).

$$
\left\{\begin{array}{l}
\dot{\boldsymbol{x}}(t)=\boldsymbol{f}(\boldsymbol{x}(t), \boldsymbol{y}(t), \boldsymbol{u}(t), \boldsymbol{p}(t)) \\
\mathbf{0}=\boldsymbol{g}(\boldsymbol{x}(t), \boldsymbol{y}(t), \boldsymbol{u}(t), \boldsymbol{p}(t))
\end{array}\right.
$$

where $\boldsymbol{x}$ is the state vector; $\boldsymbol{y}$ is the algebraic variable vector; $\boldsymbol{u}$ is the input vector, $\boldsymbol{p}$ is the parameter vector; and $\boldsymbol{f}$ and $\boldsymbol{g}$ are nonlinear vector-valued functions. For power system applications, the measurements are sampled in a discrete manner and thus, (1) needs to be discretized to be compatible with online measurements. To this end, the measurement function at time instant $k$ can be written as $\boldsymbol{z}_{k}=\boldsymbol{h}\left(\boldsymbol{x}_{k}, \boldsymbol{y}_{k}, \boldsymbol{u}_{k}, \boldsymbol{p}_{k}\right)$, where $\boldsymbol{z}_{k}$ can come from PMUs, merging units (MUs), digital fault recorders, and in general intelligent electronic devices (IEDs); and $\boldsymbol{h}$ is a nonlinear vector-valued function. The way of solving (1) using DSE can be found in [1].

For capturing the electromechanical transients, phasor representation is leveraged while fast-electromagnetic transients are neglected. Measurement vector $\boldsymbol{z}_{k}$ usually includes time-synchronized phasor measurements from PMUs, such as voltage and current phasors, the calculated real and reactive powers, frequency and rate of change of frequency (RoCoF); and the state vector $\boldsymbol{x}$ includes internal dynamic variables of synchronous machines and dynamic loads. Note that the phasor measurements provide the fundamental frequency phasor voltages and currents, while the electromagnetic transients are filtered out. Thus, the model in (1) represents the electrical quantities with phasors, and the electromechanical system and control dynamics are formulated with differential equations.

To capture fast electromagnetic transients in a power electronics-dominated power system, there is an increasing need to use SV measurements directly. The synchronized SV measurements contain rich time-domain information and can be obtained from MUs, which can be standalone devices or embedded in non-conventional instrument transformers and other apparatus. The standard SV sampling rates in MUs are 80 or 256 samples per cycle according to IEC61850-9-2LE standard [7]. Note that phasors and harmonics can be computed from SVs. Furthermore, electromagnetic transient models must be adopted to represent the fast dynamics of system components. The latter can include any components with electromagnetic transients, such as generators, transmission lines, transformers, CBRs, among others [8]. In this case, the voltages and currents are expressed using instantaneous SVs; the electromagnetic transients, such as fast electromagnetic transients of voltages or currents, are considered. Measurement vector $\boldsymbol{z}$ includes SV measurements, and the state vector $\boldsymbol{x}$ consists of instantaneous voltages, currents, generator speeds, and other internal states of the dynamic components.

The applications of DSE using PMU measurements for electromechanical transients include both control and protection. For control applications, DSE contributes to the enhancement of the visibility of system operations, and the validation and calibration of the control models. Furthermore, it provides essential feedback state signals and accurate measurements for controls. It is shown in the literature that DSE-based out-of-step 
protection provides more benefits for fast and reliable relay actions than traditional approaches.

DSE using SV measurements for both electromagnetic and electromechanical transients also enable advanced control and protection applications. For the control applications, it can 1) provide essential state feedback signals for CBR control, including both grid following and grid forming controls to enhance system stability; 2) identify unknown parameters and help calibrate electromagnetic models; 3 ) detect and diagnose anomalies locally, especially for converter-interfaced resources; and 4) provide accurate frequency measurements even in the presence of large disturbances. The widely used phase-lockloop is well-known to be vulnerable to large disturbance and can trigger erroneous relay actions, for example, see the 2016 California blue-cut fire events [9]. For protection applications, it allows us to 1) design protective relays with improved dependability, security, sensitivity, and speed; 2) develop fault locators that work with short data window with improved fault location accuracy; and 3) enable cyber intrusion/hidden failure detection of protective relays. These issues will be discussed in subsequent sections.

\section{CONTROLler Options: DSE VERSUS OBSERVER}

Both observers and DSE can be used to provide state feedback signals for control. In this section, the advantages and disadvantages of these two methods are discussed. Note that the detailed formulations of observers and DSE can be found in [1] and [13].

Operational principle: Observers are based on sensor outputs measured from the physical system and are usually based on the Luenberger criterion [13]. The sliding mode observer and the observer using the Koopman-based model of the process are also proposed [14]. DSE is based on the minimum variance estimation criterion from a statistics point of view and it fuses both the physical model predictions and the sensor outputs. Although these two approaches fundamentally differ in their assumptions and algorithmic details, both can be used to estimate the state of a dynamic system.

Addressing stochastic systems: The majority of designed observers in the literature do not assume statistical distributions for the process and measurement models. Most control-theoretic observer designs assume the existence of unknown inputs and sensor noise in a deterministic way without associating any distribution to these uncertainties. This is because deterministic observers still perform well in the presence of various noise distribution types. Note that gain matrix design for an observer can be challenging for large nonlinear systems due to high nonlinearity in state transition [15]. A good observer design can provide robustness to exogenous disturbances, though its performance cannot adapt to time-varying changes unless it evolves with time. Furthermore, if the noise distribution deviates from assumptions, such as Gaussian distribution, an observer may obtain better performances than the traditional DSEs using Kalman filters since an observer only requires the bounds for noise. During information extraction from data, there are random fluctuations or measurement errors in the data that are not subject to any models. They are called stochastic noises. When the phenomenon being modeled is too complex and some approximations are applied, errors are induced and they are called deterministic noises or biases (i.e., model uncertainty/error). Both DSE and observer need to be designed to handle both types of noises. The comparisons of robust DSE and observer considering different types of noises, i.e., both deterministic and stochastic need further investigations.

Sensitivity to outliers: Measurements are frequently subjected to outliers and the model outputs can be corrupted by gross errors due to control failures, incorrect model inputs, parameter errors, etc. [16]. In the presence of outliers, both state observer and DSE may provide biased results. To mitigate the influence of outliers, an observer needs to increase the assumed error bounds, which would significantly decrease its performance in the absence of outliers. In contrast, by adopting a statistical test or developing robust DSEs, outliers can be automatically detected and suppressed without affecting DSE performance when there are no outliers [1] [16].

Computational efficiency: The majority of DSE designs recursively compute gains; this involves matrix multiplications and inversions at each time-step. In contrast, once the observer is designed offline, state estimation can be performed with a significantly smaller number of matrix multiplications, as the calculations of observer gain are usually done offline assuming certain error bounds of process and measurement models [17]. This offline calculation involves computationally costly linear matrix inequalities and convex semidefinite programs. Thus, recalculation of observer gains is time-consuming in the presence of changes in the network topology or parameters, whereas DSE can update such new information more efficiently at each time-step.

Sensor requirements: Both DSE and observers require the system/states to be observable as a prerequisite from the dynamical system perspective [1]. For DSE, more sensors would lead to better statistical efficiency of the state estimates. Observers usually suffer from a key limitation regarding the required number of sensors, which can lead to infeasible observers [18]-[19], theoretically infinite estimation error, or practically unusable estimates. DSEs, however, still produce some useful results even with a limited number of sensors.

Handling system nonlinearity: Both DSE and observers can be designed to deal with nonlinear systems. However, designing a good observer when the system is subjected to complex nonlinearities is very challenging as it involves nonlinear optimization [19]. Also, the Jacobian matrix is needed for some nonlinear observer designs. However, magnetic saturation in synchronous machines is frequently encountered in power systems [2], making infeasible the computation of Jacobians and subsequent observer design. In contrast, there exist derivativefree nonlinear Kalman filter-based DSEs, such as unscented Kalman filter, ensemble Kalman filter, particle filter, etc. [1], which avoid the use of Jacobian matrix and thus handle system nonlinearity better.

Sensitivity to initial conditions: For DSE, a good initial condition is usually needed, otherwise, it takes some time to converge to the true value. This, in general, is not an issue since DSE runs continuously and in the long run, once it has converged, the initial condition is the state estimate of the system at the prior time step, which is an accurate initial condition. For state observers, irrespective of the initial condition, fast convergence of the estimated states to their accurate values is guaranteed as long as a proper observer gain is determined, and the system is observable. It should be noted that practically, a reasonable initial condition can typically be obtained by utilizing power flow calculations or state estimation, or engineering judgments or experiences. 


\section{Control Applications of DSE}

Overview of control: Control of different components in a power system is performed by controlling the devices which govern their power and voltages. For a synchronous generator, these devices are the governor (which controls the mechanical power input) and the automatic voltage regulator (AVR) (which controls the excitation voltage). For CBR, on the other hand, these devices are the power electronics converters, which have a few control options, for example, maximum power tracking and voltage control, real and reactive power control, real power and voltage control, and $\mathrm{AC} / \mathrm{DC}$ voltage control. In addition, depending on operating conditions, the controls can switch to low voltage ride through logic, storage control, etc. Since synchronous generator controls are slower than converter controls, DSE must provide feedback to the controllers on a commensurate time scale. DSE operating at a cycle scale can meet the requirements of controllers for synchronous machines and converter control. For other applications such as protection and associated controls, DSE needs to operate at faster time scales and typically uses SV measurements. An overall scheme for implementation of DSE-based control of power systems is shown in Fig. 1. It should be noted that the "traditional phasors" assume steady state operation of the system, while the "synchrophasors" obtained from PMUs are time synchronized phasors with fastvarying amplitudes and phase angles, which can better describe the dynamics of the system [20]. However, the synchrophasors are still based on the framework of phasors, which assumes sinusoidal-shape waveform, but with a much faster update rates (hundreds of samples per second). By contrast, SV measurements directly utilize measured values from the waveforms at each sample, with full description of measurement transients. Thus, PMU measurements and SV measurements are two options for controls with fast time scales.

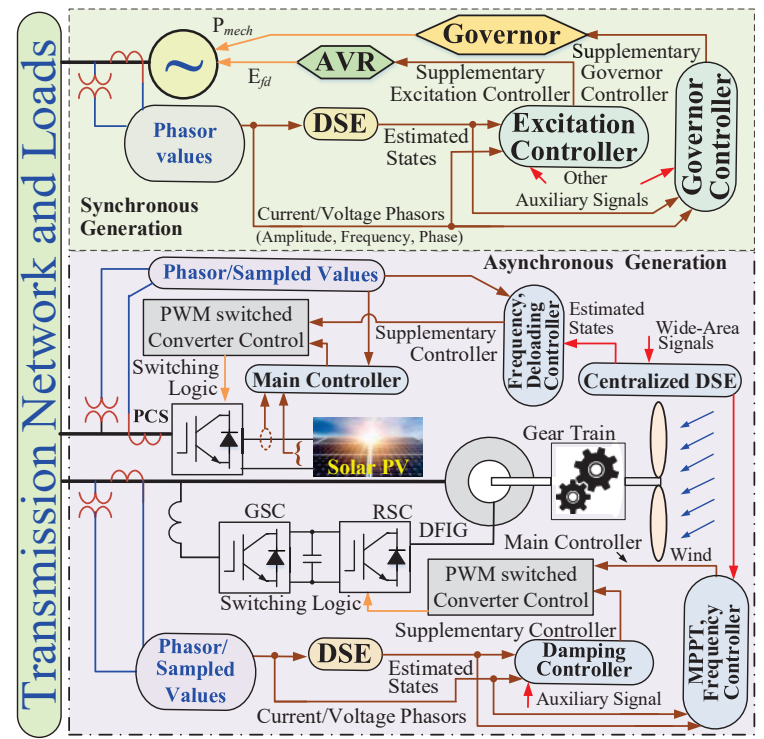

Fig. 1. Example implementation of DSE-based control architecture

\section{A. DSE-Based Control using PMU Measurements}

\section{1) DSE-based Control of Rotor Angle Stability}

As synchronous generators form the backbone of today's AC power system, they are the main points of control-actuation and can be directly controlled using excitation systems \& governors. Other important actuators are the transmission paths, controlled using FACTS devices and transformer taps. DSE-based control design can be centralized, decentralized, or hierarchical (Fig. 2).

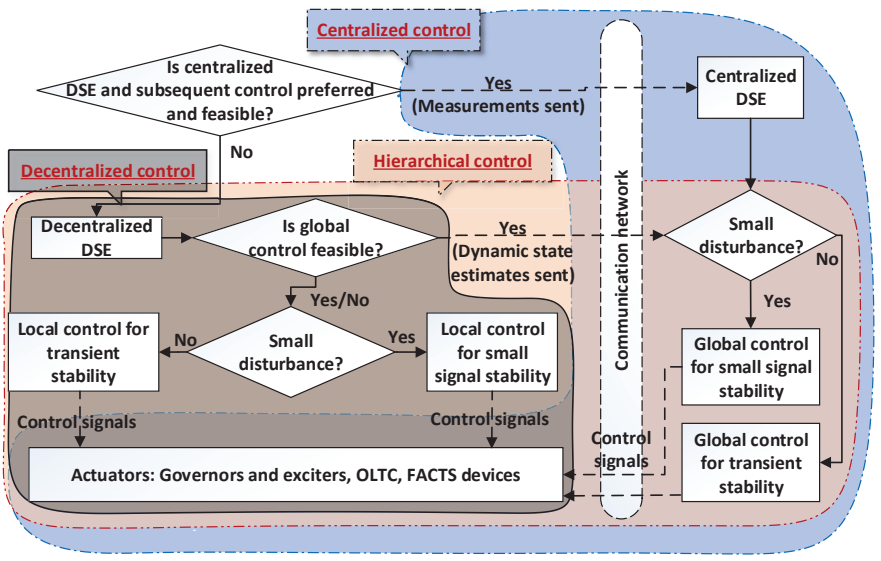

Fig. 2. A flowchart of DSE-based control decision and design process for rotor angle stability

\section{Centralized and Hierarchical Control}

In DSE-based centralized control, measurements from across the system are transmitted to a central location, and DSE for the whole system is performed [21]-[23], as shown in Fig. 2. A control law is then obtained using the system model and the dynamic state estimates via various control techniques, including $L_{\infty}$ robust control that models disturbances as $L_{\infty}$ bounded inputs and finds a state-feedback control law using nonconvex optimization [21]; linear quadratic regulator (LQR) where the quadratic costs of control and state-deviation are optimized with pole-placement for crucial system eigenvalues using state-feedback [22]; and residue-based control, where pole-placement is explicitly done using power system stabilizers (PSSs)/power oscillation dampers [23]. Some limitations of centralized control that restrict its field implementation are: communication latencies can impact performance, an accurate model of the whole system at the central location is required, and communication failures and bottlenecks can create serious issues.

A partial solution to the problems of centralized control is hierarchical control in which decentralized DSE is performed at machine locations and the estimates are sent to a central location (or PMU measurements are sent to a central location for performing DSE in a decentralized manner using a federation of estimators [24]). Global control laws are then developed at the central location along with local control laws for decentralized locations. The local and global controls form the two levels of hierarchical control, as shown in Fig. 2, and are implemented using actuators, such as FACTS devices and excitation systems of synchronous generators [25]-[26]. In the worst-case scenario, such control can also be used to shed run-away generators on the fly to prevent instability [27]. The hierarchical control approach also requires knowledge of a complete system model at the central location.

\section{Decentralized Linear Control}

In decentralized control, each generator is controlled independently, requiring only local measurements for both DSE and control. By controlling local machine dynamics, some aspects of global system dynamics may also be controlled. This eliminates communication requirements, but in many cases, it suffers from limited system-level controllability/observability. As a result, special techniques are needed to establish system stability. Both linear and nonlinear methods can be used to implement such control. 
Linear methods of DSE based decentralized control are valid only for small-signal dynamics [6], and are similar to the traditional PSSs: in PSSs the control parameters are tuned offline, while DSE-based methods do so online. Such a 'dynamic tuning' of control parameters can be done using various methods, such as decentralized $L_{\infty}$ [21], in which a convex approximation of centralized $L_{\infty}$ formulation ensures that only local machine states are needed for control; and extended LQR [28], in which the costs of voltage phasors (as exogenous inputs) are included in the LQR costs to find control gains. A drawback of linear methods is that the asymptotic stability of the whole system cannot be guaranteed. However, this is usually not an issue as linear methods are applicable only for small disturbances, which usually do not alter the asymptotic stability.

Another linear control approach is to use data-driven DSE, where local measurements are used to find a linear Koopman predictor [14] to mimic system dynamics, which is then used to perform DSE (similar to an observer) and to design model predictive control (MPC). The estimated rotor speed of each generator is used as a feedback signal in the MPC for excitation control. To build the Koopman predictor, the data of all the states and measurements are collected in matrixes $\mathrm{X}$ and $\mathrm{Y}$, respectively, for different trajectories of the system, while inputs are collected in matrix U. Lifting radial basis functions (RBFs), $\psi(x)$, are used for lifting $\mathrm{X}$ and $\mathrm{Y}$, and are defined as follows (with the centers $x_{0}$ obtained randomly from uniform distribution in $[-1,1])[14]$ :

$$
\psi(x)=\left\|x-x_{0}\right\|^{2} \cdot \log \left(\left\|x-x_{0}\right\|\right)
$$

After data lifting, extended dynamic mode decomposition is used to compute the lifted matrices, which represent the predicted model used to design the MPC controller. The lifted matrices (A, B, C) are the solution to the following optimization problem [14], with $X_{\text {lift }}$ and $Y_{\text {lift }}$ denoting the lifted states and measurements, respectively, and $U$ denoting the inputs.

$$
\min _{A, B} \sum_{J=1}^{k}\left\|Y_{\text {lift }}-A X_{\text {lift }}-B U\right\|_{2}^{2} ; \min _{C} \sum_{J=1}^{k}\left\|X_{\text {lift }}-C X_{\text {lift }}\right\|_{2}^{2}
$$

Fig. 3 shows that the proposed Koopman MPC (KMPC) provides a much better oscillation damping performance as compared to the classical PSS, after a $100 \mathrm{~ms}$ symmetrical threephase fault at the infinite bus in a single machine infinite bus system $\left(\omega_{1}\right.$ denotes the rotor speed of the machine, while $V_{t}$ denotes its terminal voltage). This example uses a highly accurate Koopman model trained with random excitations, with dimension $\mathrm{N}=108$ ( 8 physical states plus $100 \mathrm{RBFs}$ ) [14]. System trajectories are generated through simulations after applying random control signal inputs to generate the trajectories. The control input for each trajectory is selected randomly from uniform distribution in $[-1,1]$. The parameters for the two-stage lead-lag PSS with a washout filter used in this example are (parameters found using the standard root-locus method [29]): $\mathrm{K}_{\mathrm{PSS}}=5.2450$, $\mathrm{T}_{1}=0.5603, \mathrm{~T}_{2}=0.0145, \mathrm{~T}_{3}=0.6614, \mathrm{~T}_{4}=0.0415, \mathrm{~T}_{\mathrm{W}}=10$.
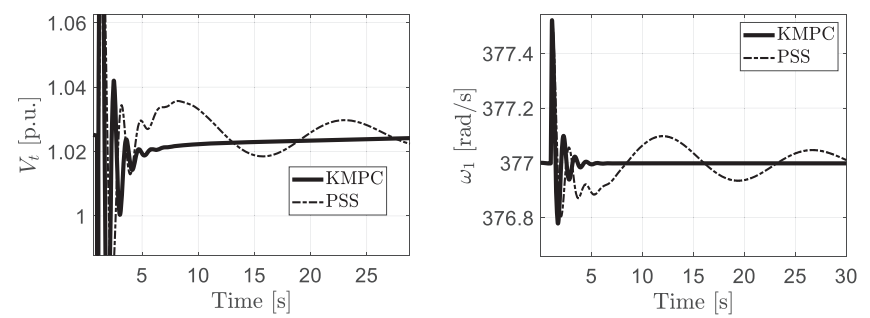

Fig. 3. Rotor speed response of KMPC vs PSS

\section{Decentralized Nonlinear Control}

Nonlinear control methods are needed to ensure the stability of the system against large disturbances, i.e. when a linear approximation of the system is no longer valid. These methods either find a partially linear transformation of system dynamics, called feedback linearization [30]-[31], or find a positive scalar with a negative time derivative, called a Lyapunov function [32]-[33]. Feedback linearization is easier to formulate and use than Lyapunov functions, but the latter has better asymptotic stability characteristics. In [30], detailed generator modeling has been used to implement feedback linearization based excitation control to ensure transient stability, while [31] considers detailed load modeling for the same. In [32], a Lyapunov function is constructed for excitation and governor control, and [33] proposes an optimal Lyapunov formulation for excitation control and uses neural networks for computational efficiency.

Although nonlinear control works well even for small disturbances, its control costs (given by the time integral of a weighted sum of the squares of control inputs) are much higher than the linear control costs. Hence, it is practical to activate nonlinear control only for large disturbances. Based on this idea, a hybrid decentralized control is proposed in which RoCoF estimation is used to infer if a large disturbance has occurred. To do this, the DSE-based RoCoF estimate obtained using [34] is multiplied with the machine's inertia to get a switching signal: if the absolute value of this signal is greater than a predefined value (say 0.5 p.u.) for two $\mathrm{AC}$ cycles, then the control is switched to the nonlinear control given in [30]. Control is switched to the linear control given in [28] only if the switching signal stays within \pm 0.5 p.u. continuously for $5 \mathrm{~s}$.

The comparison of performances of the hybrid decentralized control and classical PSS control is shown in Fig. 4. Test system model, simulation conditions, and controller parameters are same as in [30]. The test system starts from steady state, and at $\mathrm{t}=1 \mathrm{~s}$ a small disturbance takes place in form of a step load change by doubling the load on bus 53 . At $t=20$ s, a large disturbance takes place in form of a solid three phase fault on bus 54 , which is cleared after $200 \mathrm{~ms}$ by opening of circuit breakers on the line 54-53. The difference between rotor speeds of machines 1 and $16\left(\omega_{1}-\omega_{16}\right)$ has been plotted in Fig. 4 corresponding to the two control methods. It can be seen that hybrid control can ensure both small-signal stability and transient stability of the system (Fig. 4), and exhibits both linear and nonlinear control properties.

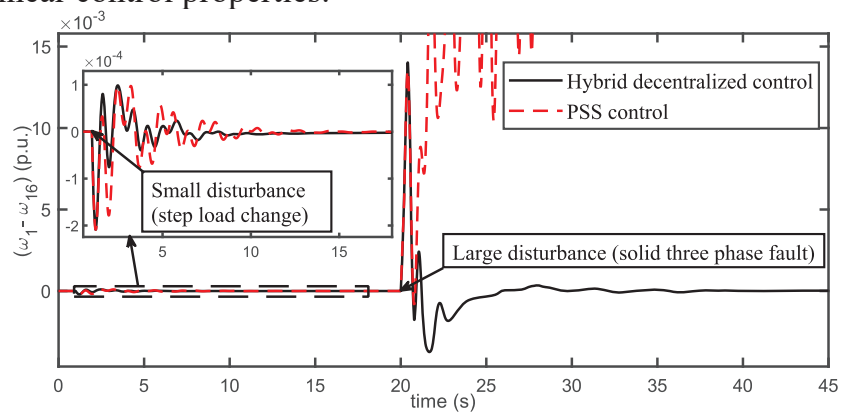

Fig. 4. Control performance of decentralized hybrid-control

\section{2) DSE-based Control of Frequency Stability}

Unpredictable load fluctuations, intermittency due to stochastic generation, and random system disturbances may result in continuous fluctuations in system frequency and tie-linepower exchanges causing them to deviate from their nominal values. These variations are corrected by governor-based load frequency controller (LFC) action [35]. The performance of 
LFCs is significantly affected by the inherent governor deadband (GDB) and generation rate constraint (GRC) [36]-[38]. In contrast, DSE-enabled LFC [39] (Fig. 1) is not affected by the GDB or GRC and uses PMU measurements to derive the auxiliary signal for the governor loop. However, communication impediments hinder the applicability of such LFCs. To address this issue, it is proposed that traditional LFCs should be assisted by a supplementary stabilizer, in which turbine-governor dynamics are estimated using decentralized DSE to derive the control signal. This improves the frequency stability margins compared to observer-based conventional LFCs (as in [40]) and is illustrated in Fig. 5.

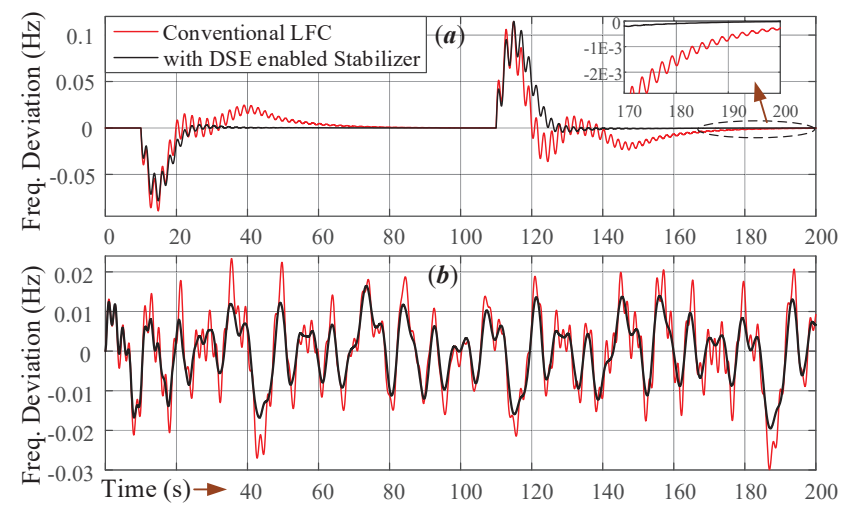

Fig. 5. Frequency Stability: DSE-Stabilizer vs conventional LFC

Fig. 5.(a) depicts the frequency response in Area 1 of the IEEE 10 machine 39 bus system (system details are as in [41]) following a stepped load increment of $2 \%$ of load at Bus 15 at $\mathrm{t}=10 \mathrm{~s}$ followed by a decrement of $2.5 \%$ at $\mathrm{t}=110 \mathrm{~s}$. Fig. 5.(b) depicts the frequency response for the system under fluctuating wind power output which was simulated by considering a realistic wind speed profile (please see [37] and [38] for the details of wind modelling). The details of turbine and governor model, including nonlinearities like GDB and GRC, are given in Appendix of [36]. The auxiliary frequency control law uses turbine states (valve opening, turbine power and reheater output), which are estimated through DSE [36], as inputs to derive the corrective control signal. The gain vector for this control signal is obtained by modelling the stochastic generation and load perturbations as unmatched perturbations and solving the resulting nonlinear equation following the same procedure as detailed in [42]. Since the time evolution of the governor states is relatively slow, the estimated states need to be supplied to the auxiliary stabilizer at a slower rate $(10 \mathrm{~Hz}$ or less) compared to reporting rate of the PMUs ( $100 \mathrm{~Hz}$ or above) using a zero-order hold.

The response in the Fig. 5.(a) illustrates that the stabilization time of the frequency improves by many fold (about 3 times) via the DSE-enabled supplementary stabilizer in the event of stepped load changes. In the event of continuous parity between generation and load due to stochastic nature of renewables, the frequency excursions are reduced significantly by employing the DSE enabled stabilizer compared to traditional LFC as illustrated by Fig. 5.(b). Therefore, DSE-enabled frequency stabilizers can help in improving the frequency control indices manifold.

\section{3) DSE-based Control of Voltage Stability}

Real-time voltage instability detection and control methods that are the best candidates to take advantage of DSE are those that use snapshots of evolving system trajectories, captured either by dedicated measurement/communication system or by a tracking state estimator [43]-[45]. In principle, those methods can use the system snapshots provided by a DSE giving access, for instance, to the excitation system status of generators (namely: is the generator controlling its voltage or is it under field or armature current limit?) and system frequency. The same holds for other voltage controlling components such as static VAr compensators or STATCOMS. Long-term voltage instability detection and control can use originally proposed approaches (like sensitivities in [43] and modal analysis in [45]). In addition to the dynamic states, the network state and topology are required to monitor, detect, and control voltage instability. In this respect, the local DSE approaches discussed in [1] are combined with network state estimation, which can be fully based on PMUs or multi-rate measurements [44]. In principle, for long-term voltage instability, the system state can be updated at low rates (less than 10 times per second). One of the advantages of DSE is its ability to provide full state estimates, thus enabling full-state feedback MPC controllers [46].

An example of emergency control against long-term voltage instabilities [47], which can be adapted to take advantage of DSE outputs and be used for long-term instability detection, is illustrated in Fig. 6.

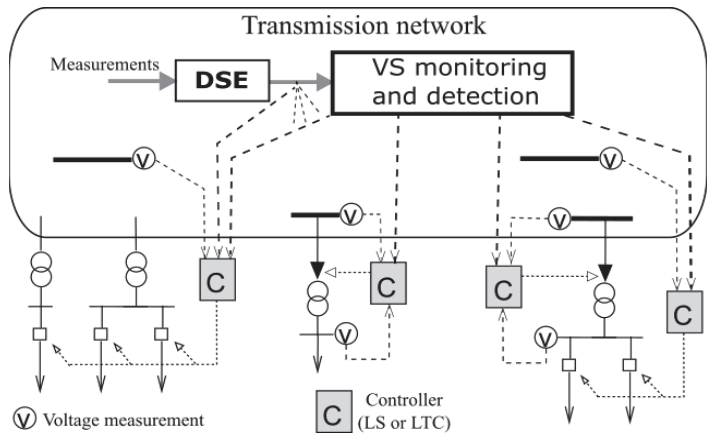

Fig. 6. A framework for two-level long-term voltage stability (VS) monitoring, instability detection, and emergency control

It is a two-level scheme. The upper level is in charge of widearea monitoring, while the lower level controllers provide remedial actions such as Load Shedding (LS) or modified Load Tap Changer (LTC) control. In an emergency voltage situation, the upper level relies on the voltage instability detection method of [43] (DSE can replace the dedicated PMU measurements proposed in[43]) and provides the lower-level controllers with minimum transmission voltages to maintain. Those voltages correspond to the point where long-term voltage instability is detected [47].

In terms of short-term voltage instability detection and control, the voltage control statuses of synchronous generators or compensators are crucial information. The phenomenon of concern is faster and a DSE used for this purpose must provide the system dynamic states at a high rate (10 to 60 times per second); a PMU-based network state estimation appears to be mandatory for this application.

Increased penetration of grid-connected CBRs (particularly when connected to weak grids) brings concerns to voltage instability (both long- and short-term). This requires even more rigorous metering infrastructure and DSE should use a detailed model with the full complement of state variables for the CBRs to solve voltage instability problems. 


\section{4) DSE for CBR Control}

The intermittent and asynchronous nature of variable CBRs, mainly wind and solar, poses several pressing challenges in today's energy systems research [6][10], especially for CBR-DSE and control. Several methods for DSE based control of CBRs have been proposed in the literature. A UKF-based DSE is developed to estimate doubly-fed induction generator (DFIG) flux dynamics and this allows for the development of a flux estimation based control scheme [48], which achieves better fault recovery response than traditional control methods. In [49], DSE has been used to estimate the electromechanical dynamics of a DFIG and the estimated states have been used to derive the supplementary signal for damping of electromechanical oscillations (Fig. 1). DSE-based sliding mode control for DFIGintegrated power systems is developed in [50] for maximum energy extraction and power quality enhancement. The idea is further extended to the DSE-based frequency restoration method considering solar irradiance variations [51]. DSE based control can also be used to damp sub-synchronous oscillations in seriescompensated lines with wind generation [52].

\section{B. DSE-based Control using SV Measurements}

Traditional generator controls are mostly decentralized as the local frequency and voltage information acts as a medium to bring the information of the rest of the grid to the local generator. Modern power systems are evolving with a lower inertia and more complex transients with CBRs. In this case, controls with local frequency and voltage information may not be sufficient; remote side information such as frequency, RoCoF, and waveform distortion can also help minimize the transients and prevent damage/shut-down of converters during disturbances. However, remote side information usually requires communication channels, resulting in increased cost and compromised reliability of the system. With the help of SV measurements and the accurate time-domain transmission line model, DSE can effectively estimate the voltages and currents at the remote side of the transmission line using local information only, without any physical communication channels between the two terminals of a transmission line [53]-[54]. Afterward, remote side information such as frequency, RoCoF, and waveform distortion is extracted from the estimated voltages and currents and is utilized as the input to CBR control. Fig. 7 depicts an example of a DSE-based converter control system using SV measurements.

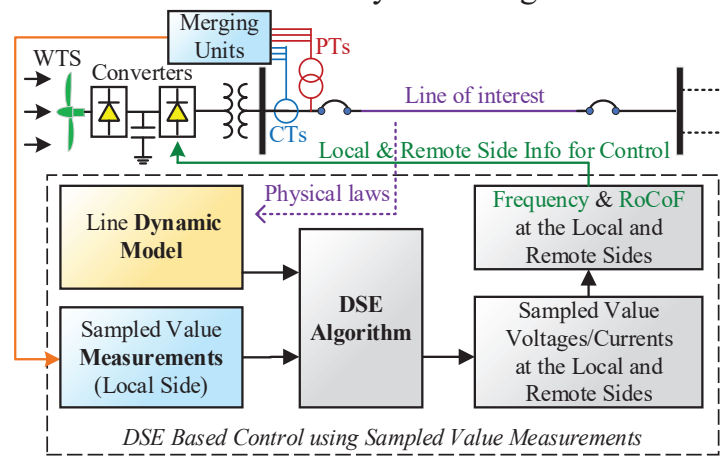

Fig. 7. DSE based converter control system using SV measurements

In this example, the local voltage and current SV measurements are first obtained via potential transformers (PTs), current transformers (CTs), and MUs. Next, using the DAEs of the line of interest, DSE estimates the dynamic states of the system, including SV voltages and currents at the remote side. Finally, the frequency and the RoCoF at the local and remote sides are extracted from the estimated SV measurements. These are fed into the local converter controller to initiate supplementary control using local and remote frequency and RoCoF to minimize transients during system oscillations and keep the converter synchronized with the system. Note that the remote side frequency information could also be approximated using phasor domain methods [55]. In addition, estimated SV states of the system can also be used for other applications such as fast control of converters, harmonics filtering, etc.

\section{PRotection APPlications of DSE}

When a fault occurs on power system components, protective relays need to immediately operate, to isolate the faulty components, minimize the power outage, and ensure the safety of human beings and the overall power system. Protective relays are evolving with improved reliability. However, statistically, the industry in the U.S. and abroad are still experiencing around 10 percent misoperation on average [56]. These are due to limited measurements, mis-coordination among relays, or faults that are hard to be quickly and reliably detected, such as high impedance faults, faults near neutrals, etc. In addition, most protective relays are based on fundamental frequency measurements, which limit their operational speed as well as applicability to DC systems. The proliferation of CBRs has also generated new challenges for legacy protection systems. Most relay settings are fixed; but with reduced system inertia, the settings need adjustment to distinguish stable and unstable swings. DSE based protection provides a new approach to deal with these challenges. DSE is a powerful tool in tracking power system transients (including electromechanical and electromagnetic transients) and therefore enables protective relays with improved operational speed, sensitivity, and reliability.

\section{A. DSE-based Protection using PMU Measurements}

1) Out-of-step Protection using Direct Stability Assessment

DSE for electromechanical dynamics has been used to monitor the stability of generators and to detect instability when it occurs. This provides better system protection for stability. Specifically, generator instability is a serious problem for power systems; generators must be protected against this condition; out-of-step (OOS) relaying is used to detect and protect the generator when it spins into instability. Present out-of-step protective relays typically monitor the apparent impedance at the terminals of a generator. When instability occurs the impedance moves from the right-hand side of the impedance diagram to the left-hand side. Upon this detection, the relay will schedule to trip the generator when the generator swings another 120 to 150 degrees, which will minimize the transients in the breaker (transient recovery voltage) and allow the reliable opening of the breaker. Because of this timetable (i.e. detection of the instability and then a delay for favorable conditions to trip the generator), the period during which the generator experiences severe current flow due to the oscillation is long.

A new DSE based protection method has been introduced to detect the onset of instability much faster than the impedancebased approach described above [57]-[58]. The basic idea is quite simple: DSE is used to estimate the full dynamic state of the generator and the immediate network to which the generator is connected. The DSE provides the speed of the generator [55], the frequency, and RoCoF at each bus of the system. A simple calculation provides the center of oscillation $(\mathrm{CoO})$. The $\mathrm{CoO}$ is characterized by the fact that the frequency at that point does 
not oscillate (it may be linearly changing with time) and the frequency at the nearest two buses oscillates with approximately 180 degrees out of phase. Knowledge of the generator speed and the location of the $\mathrm{CoO}$ enables the computation of the total energy of the generator,

$$
E_{\text {total }}=E_{p}+E_{k}=-\int_{\delta_{\text {coo }}}^{\delta}\left(P_{m}-P_{e}(x)\right) d x+\frac{1}{2} M \omega^{2}
$$

where $E_{\text {total }}, E_{p}$ and $E_{k}$ are the total energy, potential energy, and kinetic energy of the generator, respectively. $M=2 \mathrm{H} / \omega_{S}$, $H$ is the per-unit inertia constant, $\delta$ is the generator position, $\omega$ is the rotor speed, and $\omega_{s}$ is the synchronous speed.

The stability of the generator is determined by the total energy using classical Lyapunov's theory. The theory states that when the total energy reaches the value of the peak potential energy (stability barrier) the generator becomes unstable. The dynamic state estimator provides the total energy of the generator as well as the potential energy function and asserts instability when the total energy reaches the stability barrier. It turns out that the assertion of generator instability occurs before the generator has swung away from the system and therefore can be tripped immediately, avoiding severe transients of an unstable generator. Next, we present a simplified example to illustrate the performance of this method.

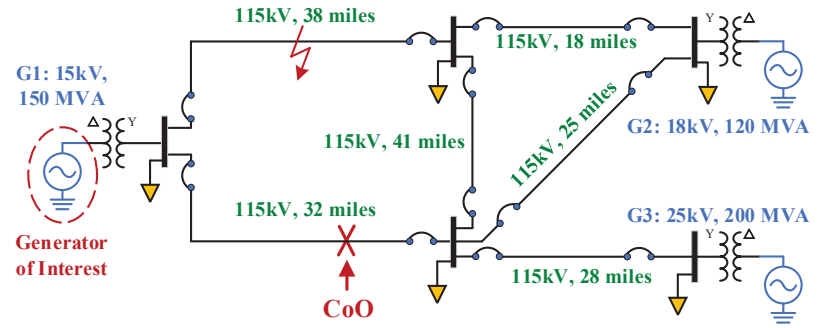

Fig. 8. Single-line diagram of the example test system

The example test system is illustrated in Fig. 8. It consists of three generators, three transformers, six transmission lines, five loads, and 12 breakers. The parameters of the generators, transformers, lines, etc. are typical. We consider a fault on the 115 $\mathrm{kV}$, a 38-mile-long line that is successfully cleared by the protection system of the line. The fault disturbs the system and oscillations are triggered.

Fig. 9 illustrates the results of the DSE and computed trajectory of the impedance of the generator as "seen" at the terminal of the generator. The trajectory is superimposed on the characteristics of an OOS legacy protective relay to compare the DSE based protection results with the legacy protection. The apparent impedance moves to a value very close to the origin upon fault initiation at time $t=1 \mathrm{sec}$. During the fault, as the generator and the system oscillate, the trajectory moves. The fault is cleared $0.25 \mathrm{sec}$ after the occurrence of the fault (i.e. $t=1.25 \mathrm{sec}$ ) by disconnecting the faulted transmission line. For this system, the critical clearing time is $0.2 \mathrm{sec}$. This means that the generator enters instability at time $\mathrm{t}=1.2 \mathrm{sec}$. The legacy relay will alarm the condition at time $\mathrm{t}=1.43 \mathrm{sec}$ and will assert instability at $\mathrm{t}=1.51 \mathrm{sec}$ while the generator rotor is at 216.2 degrees. At this angle, the generator cannot be tripped immediately (due to concerns over transient recovery voltage at the generator breaker and possibility of restrike) but need to wait until the phase angle goes to a smaller value. The DSE estimates the potential energy after the clearing of the fault and due to computational latencies, it asserts instability at $\mathrm{t}=1.29 \mathrm{sec}$ when the generator rotor is at 118.4 degrees out of phase with the center of oscillation. At this phase angle, the generator can be tripped immediately, avoiding any additional stress on the generator.

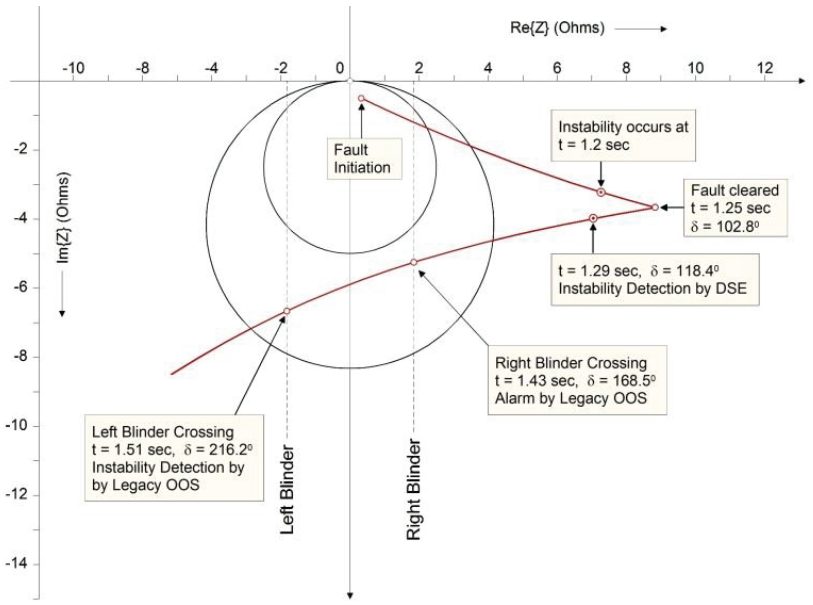

Fig. 9. Instability assertion time via DSE and comparison to legacy OOS Protection

2) Adaptive OOS Relay Settings Approach

Adaptive OOS settings based on dynamic model parameters estimation preserves existing industrial practice, and it is assisted by DSE for electromechanical dynamics. The OOS settings are mainly influenced by the direct axis transient reactance, quadrature axis speed voltage, and generator inertia. As seen by the relay, these parameters vary with time in the modern grid architecture with high penetration of power electronic interfaced technologies. The approach reported in [59] can be used to provide this real-time information for OOS relay settings recalculation. DSE is used to estimate dynamic model parameters which are used to recalculate the setting of OOS to adapt the relay sensitivity towards the generator instability with the system operating condition before the disturbance. This approach has been reported in [60]. In the paper, the settings of OOS are recalculated based on the extended equal area criterion method and demonstrated to be more effective OOS condition detection and protection.

\section{B. DSE-based Protection and Fault Location Using SV meas- urements}

\section{1) DSE-based Protection Using SV Measurements}

Compared to the synchrophasor measurements from PMUs, synchronized SV measurements from state-of-the-art MUs capture system dynamics in time domain. DSE can be utilized to extract the system information embedded in SV measurements, to provide reliable detection of fault conditions that are not reflected by fault current levels, distortion of waveforms and characteristics of fault currents. In addition, the use of SV measurements provides detection of faulty conditions much faster than legacy protection functions (such as overcurrent protection, distance protection, current differential protection, etc.), which usually require collection of enough data to compute phasors, resulting in fault detection delays.

DSE based protection methods using SV measurements have been introduced in [8]. These methods were inspired from the widely adopted current differential protection. The latter examines whether the phasor domain KCLs of the protection zone is satisfied; many but not all internal faults will violate KCL allowing differential protection to detect these faults and trip the component. By contrast, DSE based protection examines whether all the physical laws of the specific protection zone are satisfied and an internal fault is detected with any violation of any physical law. Depending on the protection zone, physical laws may include KCLs, KVLs, motion laws, thermodynamic 
laws, etc. The primary improvements of the DSE based protection compared to the existing protection approaches (such as current differential protection) include: 1) speed: DSE based protection approach utilizes time-domain SV measurements instead of phasor/spectral domain synchrophasor measurements, which enables faster fault detection; 2) applicability to DC systems; 3) DSE based protection approach checks all the physical laws (instead of KCLs only), and therefore can detect internal faults with improved sensitivity and reliability. Note that current differential protection fails to detect some faults, for example, inter-turn faults in transformers, etc.

A systematic procedure for DSE-based protection is as follows: 1) building the dynamic model that encapsulates all the physical laws of the protection zone in the time domain; the model uses differential and algebraic equations (DAEs) that could include electromechanical, electromagnetic, and thermal transients; the model represents a high fidelity time-domain representation of the protection zone; 2) applying DSE for estimating dynamic states and checking the consistency between the available measurements and the dynamic model. Low consistency indicates that some of the physical laws are violated and therefore an internal fault is detected. The validity of the DSE based protection comes from the following key advantages of DSE: 1) accurately tracking the dynamics of the system; 2) systematically checking the consistency between the measurements and the dynamic model through the residuals, and 3 ) effectively filtering out measurement errors. DSE based protection schemes have been applied to transmission lines [61]-[62], microgrids [54], transformers [63], etc. With increased security and dependability compared to legacy methods, the DSE based protection can be utilized as the main protection of the component of interest (protection zone). Additionally, the DSE based protection is capable of detecting bad data through centralized substation protection (details in section V.B.3).

The implementation of an example DSE based protective relay with a two-terminal transmission line as the protection zone is shown in Fig. 10. Due to space limitations, the figure only demonstrates the relay on the left terminal of the line, with the inter-trip signal connected to the left side breaker (the relay on the right terminal is equivalent).

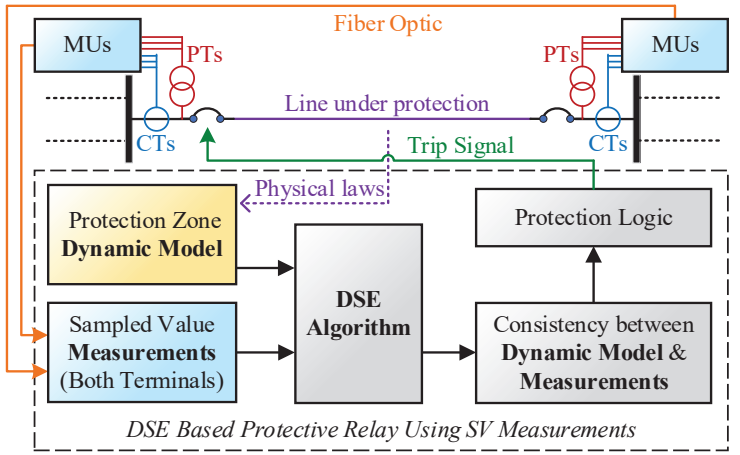

Fig. 10. Example DSE based protective relay using SV measurements at the left terminal, with a 2-terminal line as the protection zone

The two-terminal voltage and current SV measurements are obtained through PTs, CTs, and the MUs. The dynamic model of the protection zone is established by describing all the physical laws of the line under protection using DAEs. Afterward, DSE combines the dynamic model and the SV measurements, solves the dynamic states of the system, and computes the consistency between the dynamic model and the measurements (via the well-known chi-square test). Finally, the trip signal is issued to open the circuit breaker of the transmission line if an internal fault is detected. Using such an approach high impedance, or arcing faults are easily detected. A similar approach can be used to design smart auto-reclosure procedures [64].

\section{2) DSE-based Fault Location Using SV Measurements}

Fault Location is another important function when the fault occurs in a protection zone. Specifically, the exact fault location should be determined to minimize the time spent searching for the fault location by repair crews, yielding reduced power outage time and operating costs. With the development of fast-tripping techniques of protective relays, the time window of available measurements during faults for fault location is further shortened to the order of milliseconds. In addition, during this short time window, the available measurements usually experience severe transients. This leads to compromised accuracy of calculated phasors and therefore increases the fault location errors for legacy phasor domain-based fault location approaches.

To deal with these challenges, DSE based fault location approaches using SV measurements have been proposed in [65][67]. They were inspired by the widely adopted traditional phasor domain fault location methods. Traditional phasor domain methods build the relationship between the available phasor domain measurements and the location of the fault using algebraic equations, which are afterward solved to identify the location of the fault. DSE based fault location methods first describe the relationship between the available time-domain measurements and the location using an accurate time-domain dynamic model of the transmission line with fault. The timedomain model can also include the model of the arc [68]. The dynamic model is a set of DAEs, which typically include instantaneous voltages and currents through the transmission line as dynamic states of the system, and also the location of the fault as an extended state. Then, DSE is applied to systematically estimate the location of the fault. The primary advantages of DSE based fault location approach over traditional phasor domain approaches are 1) the DSE based methods make full use of information embedded in SV measurements and systematically filter out measurement errors; 2) time-domain algorithms typically possess faster convergence compared to phasor domain algorithms; 3 ) time-domain algorithms are not sensitive to complex harmonics distortions and are capable of accurate tracking severe dynamics during faults [68].

3) Hidden Failure Detection of Protective Relays using DSE

DSE-based protection schemes have also been applied at the substation level to achieve centralized substation protection [69], to detect compromised devices such as MUs and relays. The main idea is to utilize all the measurements in a substation, resulting in high data redundancy; this enables surgical detection of bad/missing data and validation of the source of anomalies (i.e. actual fault in the system, instrumentation faults, bad data injection cyber-attacks, etc.) via systematic hypothesis testing. Instrumentation faults (blown fuses, shorted CT, incorrect instrumental transformer ratios, etc.) known as hidden failures are a real problem in any protection system. DSE offers another advantage: upon detecting a hidden failure and bad data, the compromised data can be replaced in real-time by estimated values to ensure the resilient and reliable operation of the protection system.

\section{CONCLUSIONS AND FUTURE WORK}

This paper has explored the usefulness and the advantages of DSE on many control and protection applications for modern power systems. It has been shown how DSE-based solutions 
comprehensively respond to challenges in the control and protection of modern power systems holistically. In addition, several gaps in the existing literature have also been identified and several new DSE-based control and protection methods have been proposed as possible solutions.

Future research on DSE applications on control and protection are categorized into the following key areas:

- Role of $\mathbf{A I} /$ machine learning: DSE enables the validation and calibration of system dynamic models, but there are still scenarios that good calibrations cannot be achieved due to the lack of high-quality large disturbances. As a result, the dynamic model may create deficiencies, yielding unreliable control, and protection. By mining the historical operational characteristics, advanced AI and machine learning tools may be able to compensate for these deficiencies. This further allows us to develop deep reinforcement learning or advanced MPCbased control algorithms that interact with the compensated DSE model outputs for system protection, control, and stability enhancement.

- Enhancement of DSE and Observer: Although the advantages and disadvantages of observer and DSE have been discussed, there is still a need to develop computationally efficient and robust alternatives in terms of handling sensor quality, model uncertainties, and nonlinearities. The practical implementation challenges for them need to be further investigated as well when designing state-feedback controls.

- Management of distributed energy resources (DERs): In cases where fully decentralized DSE-based protection and control for all DERs do not cover all protection and control needs, as in cases of grid forming converters, multi-agent distributed DSE-based approaches need to be investigated, so as to share and transmit key information for frequency and voltage control, and protective actions.

- DSE based control for inertia emulation: One possible solution for the decreasing system inertia due to increase in CBRs can be through estimation of the states as well as the frequency, rate of change of frequency of the CBRs using DSE. These estimates can be utilized to control the power output of the CBRs in an intelligent manner such that the CBRs can provide inertial response to the grid and, to some extent, also participate in its frequency control.

- DSE based protection with high penetration of CBRs: For systems with high penetration of CBRs, protection systems encounter additional challenges with the extremely fast electromagnetic transients. Therefore, DSE tools should be carefully validated to ensure their capability of tracking electromagnetic transients in those conditions. In addition, parameter identification of CBRs using DSE can also improve the visibility of the system and therefore benefit the protection design. Moreover, the computational complexity of DSE should be considered to ensure real-time protection operation.

- DSE-based optimal operations of distributed microgrid: DSE has already been used for estimating and controlling solar power sources, wind energy power sources, traditional generation units, and monitoring and estimation of batteries. Aided by MPC and data-driven methods, the applications of DSE can be extended to microgirds stability and operation, synergistically achieving optimal operations of renewable energy microgrids. Overall, this will help supply electricity in a economic, reliable and sustainable manner.

- Design of fault tolerant and reconfigurable controls: It is reasonable to expect that DSE become a key enabler to design fault tolerant and reconfigurable controls particularly for long-term phenomena (like long-term voltage instability). A good starting point is to take advantage of similar and proven approaches from other engineering fields [70]. The ability of DSEs to detect and handle bad measurements (failure) and topology changes (errors in the model) would further simplify this problem focusing on control failures.

- DSE enabled control of energy storage state-of-charge for grid scale applications: Reliability, longevity, and efficiency of electrochemical energy storage systems like battery storage deployed for grid scale applications, such as intermittency mitigation, frequency control etc., can be enhanced by utilizing its internal states like state-of-charge $(\mathrm{SoC})$ in modulating the flow of energy from/to the energy storage via control of its power conditioning circuit. DSE can utilize the terminal measurements of the energy storage unit to dynamically estimate the SoC (otherwise unmeasurable directly) to ensure optimal flow of energy from/to the energy storage system while keeping overcharge/over-discharge in check to avoid its degradation thereby prolong its life expectancy.

- Practical implementation: Practical implementations should address issues of response time commensurate with the application. DSE applications need to be supported by adequate computing resources or distributed to achieve practical and acceptable performance in real-time. Many DSE applications of control and protection lack examples of their practical implementation in the field. Research topics include: How to ensure interoperability with currently used tools of control and protection? How to make them compatible with the current control room environment? What level of centralized or distributed computing is needed? What response times are desirable? What information should be communicated between remote sites and control rooms? What training should be developed and provided to power engineers? And what new standards are needed?

\section{ACKNOWLEDGMENT}

The authors would like to thank Dr. Yingchen Zhang, Dr. Shahrokh Akhlaghi, and Dr. Anjan Bose for their valuable discussions.

\section{REFERENCES}

[1] J. Zhao, et al., "Power system dynamic state estimation: motivations, definitions, methodologies, and future work," IEEE Trans. Power Syst., vol. 34, no. 4, pp. 3188-3198, July 2019.

[2] J. Zhao, et al., "Roles of dynamic state estimation in power system modeling, monitoring and operation," IEEE Trans. Power Syst., 2020.

[3] N. Hatziargyriou et al., "Definition and classification of power system stability revisited \& extended," IEEE Trans. Power Syst., 2020.

[4] V. Telukunta, J. Pradhan, A. Agrawal, M. Singh and S. G. Srivani, "Protection challenges under bulk penetration of renewable energy resources in power systems: A review", CSEE Journal Power Energy Syst., vol. 3, no. 4, pp. 365-379, Dec. 2017.

[5] Protection System Misoperation Task Force, "Misoperations report”, NERC Planning Committee, Atlanta, GA, USA, 2014.

[6] "Stability definitions and characterization of dynamic behavior in systems with high penetration of power electronic interfaced technologies," IEEE Technical Report, IEEE Power and Energy Society, Power System Dynamic Performance Committee, 2020.

[7] IEC Std 61850, "Communication Networks and Systems in Substations", 2003. 
[8] A. P. Meliopoulos et al., "Dynamic state estimation-based protection: status and promise," IEEE Trans. Power Delivery, vol. 32, no. 1, pp. 320-330, Feb. 2017.

[9] "1,200 MW Fault Induced Solar Photovoltaic Resource Interruption Disturbance Report: Southern California 8/16/2016 Event”, NERC, Atlanta, GA, June 2017.

[10] Z. Huang, H. Krishnaswami, G. Yuan, and R. Huang, "Ubiquitous power electronics in future power systems", IEEE Electrification Magzine, September 2020.

[11] V. Venkatasubramanian, H. Schattler, and J. Zaborszky, "Fast time-varying phasor analysis in the balanced three-phase large electric power system," IEEE Trans. on Automatic Control, vol. 40, no. 11, pp. 1975-1982, Nov. 1995.

[12] S. R. Sanders, J. M. Noworolski, X. Z. Liu, G. C. Verghese, "Generalized averaging method for power conversion circuits," IEEE Trans. on Power Elec., vol. 6, no. 3, pp. 251-259, April 1991.

[13] H. K. Khalil, Nonlinear Systems, 3rd ed., Prentice-Hall, 2002.

[14] M. Korda and I. Mezić, "Linear predictors for nonlinear dynamical systems: Koopman operator meets model predictive control," Automatica, vol. 93, pp. 149-160, Jul. 2018.

[15] M. Nicolai, L. Lorenz-Meyer, A. Bobtsov, R. Ortega, N. Nikolaev, and J. Chiffer, "PMU-based decentralized mixed algebraic and dynamic state observation in multi-machine power systems", IET Gener. Transm. Distrib., accepted.

[16] J. B. Zhao, L. Mili, "A robust generalized-maximum likelihood unscented Kalman filter for power system dynamic state estimation," IEEE Journal of Selected Topics in Signal Processing, vol. 12 , no. 4, pp. 578-592, 2018.

[17] J. Qi, A.F. Taha, J. Wang, "Comparing Kalman filters and observers for power system dynamic state estimation with model uncertainty and malicious cyber-attacks," IEEE Access, vol. 6, pp. 77155-77168, 2018.

[18] S. Nugroho, A.F. Taha, J. Qi, "Robust dynamic state estimation of synchronous machines with asymptotic state estimation error performance guarantees," IEEE Trans. Power Syst., vol. 35, no. 3, pp. 1923-1935, 2020.

[19] S. Stefani, H. Savant, Design of Feedback Systems, Oxford University Press, New York, 2002

[20] J. A. de la O Serna, "Dynamic phasor estimates for power system oscillations," IEEE Trans. Instrumentation and Measurement, vol. 56, no. 5, pp. 1648-1657, Oct. 2007.

[21] A.F. Taha, M. Bazrafshan, S. A. Nugroho, N. Gatsis, J. Qi, "Robust control for renewable-integrated power networks considering input bound constraints and worst-case uncertainty measure," IEEE Trans. Contr. Network Syst., 2019

[22] A. K. Singh, R. Singh, B.C. Pal, "Stability analysis of networked control in smart grids," IEEE Trans. Smart Grid, vol. 6, no. 1, pp. 381-390, Jan. 2015.

[23] A.M. Ersdal, L. Imsland, K. Uhlen, "Model predictive load frequency control," IEEE Trans. Power Syst., vol. 31, no. 1, pp. 777785, Jan. 2016.

[24] A. Paul, I. Kamwa and G. Jóos, "Centralized Dynamic State Estimation Using a Federation of Extended Kalman Filters With Intermittent PMU Data From Generator Terminals," IEEE Trans. Power Syst., vol. 33, no. 6, pp. 6109-6119, Nov. 2018.

[25] E. Ghahremani, I. Kamwa, "Local and wide-area PMU-based decentralized DSE in multi-machine power systems," IEEE Trans. Power Syst., vol. 31, no. 1, pp. 547-562, 2016.

[26] W. Yao, L. Jiang, J. Wen, Q. H. Wu, S. Cheng, "Wide-area damping controller of FACTS devices for inter-area oscillations considering communication time delays," IEEE Trans. Power Syst., vol. 29, no. 1, pp. 318-329, Jan. 2014.

[27] A. Paul, I. Kamwa, G. Joos, "PMU signals responses-based RAS for instability mitigation through on-the fly identification and shedding of the run-away generators," IEEE Trans. Power Syst., vol. 35, no. 3, pp. 1707-1717, May 2020.

[28] A. K. Singh, B. C. Pal, "Decentralized control of oscillatory dynamics in power systems using an extended LQR," IEEE Trans. Power Syst., vol. 31, no. 3, pp. 1715-1728, May 2016.

[29] M.A. Pai, P.W. Sauer, Power System Dynamics and Stability,
Prentice Hall, New Jersey, USA, 1998.

[30] A. K. Singh, B. C. Pal, "Decentralized nonlinear control for power systems using normal forms and detailed models," IEEE Trans. Power Syst., vol. 33, no. 2, pp. 1160-1172, Mar. 2018.

[31] S. Liu, X. Li, D. Chen, "Wide-area-signals-based nonlinear excitation control in multi-machine power systems", IEEJ Trans. Elec. Electron. Eng., vol. 14, pp. 366-375, 2019.

[32] H. Liu, J. Su, J. Qi, N. Wang, C. Li, "Decentralized voltage and power control of multi-machine power systems with global asymptotic stability," IEEE Access, vol. 7, pp. 14273-14282, 2019.

[33] A. S. Mir, S. Bhasin, N. Senroy, "Decentralized nonlinear adaptive optimal control scheme for enhancement of power system stability," IEEE Trans. Power. Syst, vol. 35, no. 2, pp. 1400-1410, 2020.

[34] A. K. Singh, B. C. Pal, "Rate of change of frequency estimation for power systems using interpolated DFT and Kalman filter," IEEE Trans. Power Syst., vol. 34, no. 4, pp. 2509-2517, 2019.

[35] H. Bevrani, Robust Power System Frequency Control. New York, NY, USA: Springer, 2009.

[36] P. Bhui, N. Senroy, A. K. Singh and B. C. Pal, "Estimation of inherent governor dead-band and regulation using unscented Kalman filter", IEEE Trans. Power Syst., vol. 33, no. 4, pp. 35463558,2018 .

[37] C. Nichita, D. Luca, B. Dakyo and E. Ceanga, "Large band simulation of the wind speed for real time wind turbine simulators," IEEE Trans. Energy Convers., vol. 17, no. 4, pp. 523-529, Dec. 2002.

[38] A. S. Mir and N. Senroy, "Self-tuning neural predictive control scheme for ultra-battery to emulate a virtual synchronous machine in autonomous power systems," IEEE Trans. Neural Netw. Learn. Syst., vol. 31, no. 1, pp. 136-147, Jan. 2020.

[39] K. Emami, T. Fernando, H. H. Iu, B. D. Nener, K. P. Wong, “Application of unscented transform in frequency control of a complex power system using noisy PMU data" IEEE Trans. Ind. Informat., vol. 12, no. 2, pp. 853-863, 2016.

[40] H. Trinh, T. Fernando, H. H. C. Iu, K. P. Wong, "Quasi-decentralized functional observers for the LFC of interconnected power systems", IEEE Trans. Power Syst., vol. 28, no. 3, pp. 3513-3514, 2013.

[41] H. Bevrani, P. R. Daneshmand, P. Babahajyani, Y. Mitani and T. Hiyama, "Intelligent LFC concerning high penetration of wind power: synthesis and real-time application," IEEE Trans. Sustain. Energy, vol. 5, no. 2, pp. 655-662, April 2014.

[42] X. Yang, H. He and X. Zhong, "Adaptive dynamic programming for robust regulation and its application to power systems," IEEE Trans. Ind. Electron., vol. 65, no. 7, pp. 5722-5732, July 2018.

[43] M. Glavic, T. Van Cutsem, "Wide-area detection on voltage instability from synchronized phasor measurements. Part I: Principle," IEEE Trans. Power Syst., vol. 24, no. 3, pp. 1408-1416, 2009.

[44] B. A. Alcaide-Moreno, C. R. Fuerte-Esquivel, M. Glavic, T. Van Cutsem, "Electric power network state tracking from multirate measurements," IEEE Trans. Instrumentation and Measurement, vol. 67, no. 1, pp. 33-44, 2018.

[45] G. K. Morison, B. Gao, P. Kundur, "Voltage stability analysis using static and dynamic approaches," IEEE Trans. Power Syst., vol. 8, no. 3, pp. 1159-1171, 1993.

[46] M. Glavic, T. Van Cutsem, "Some reflections on model predictive control of transmission voltages," 38th North American Power Symposium, Carbondale, IL, USA, 2006.

[47] B. Otomega, M. Glavic, T. Van Cutsem, "A two-level emergency control scheme against power system voltage instability," Control Engineering Practice, vol. 30, pp. 93-104, 2014.

[48] S. Yu, T. Fernando, K. Emami and H. H.-C. Iu, "Dynamic state estimation-based control strategy for DFIG wind turbine connected to complex power systems," IEEE Trans. Power Syst., vol. 32, no. 2, pp. 1272-1281, 2016.

[49] A. S. Mir and N. Senroy, "DFIG damping controller design using robust CKF based adaptive dynamic programming," IEEE Trans. Sustain. Energy, vol. 11, no. 2, pp. 839-850, Apr. 2020.

[50] S. Yu, G. Zhang, T. Fernando and H. H.-C. Iu, "A DSE-based 
SMC method of sensorless DFIG wind turbines connected to power grids for energy extraction and power quality enhancement," IEEE Access, no. 6, pp. 76596-76605, 2018.

[51] S. Yu, L. Zhang, H. H. Lu, T. Fernando, K. P. Wong, "A DSEbased power system frequency restoration strategy for PVintegrated power systems considering solar irradiance variations," IEEE Trans. Industrial Informatics, vol. 13, no. 5, pp. 2511-2518, 2017.

[52] "Wind Energy Systems Subsynchronous Oscillations: Events and Modeling," IEEE PES Wind SSO Taskforce, Technical Report, PES-TR80, 2020.

[53] A. P. Meliopoulos, V. Vittal, M. Saeedifard, and R. Data, "Stability, protection and control of systems with high penetration of converter interfaced generation", PSERC Publication 16-03, March 2016.

[54] Y. Liu, A. P. Meliopoulos, L. Sun, and S. Choi, "Protection and control of microgrids using dynamic state estimation", Protection Control Modern Power Syst., vol. 3, no. 31, pp. 1-13, Oct. 2018.

[55] R. Grondin A. Heniche, et al., "Loss of synchronism detection a strategic function for power system protection", Proc. 2006 CIGRE Session 41 B5-205.

[56] North American Electric Reliability Corporation, "State of Reliability 2016", May 2016.

[57] E. Farantatos, R. Huang, G. J. Cokkinides, A. P. Meliopoulos, “A predictive generator out-of-step protection and transient stability monitoring scheme enabled by a distributed dynamic state estimator," IEEE Trans. Power Del., vol. 31, no. 4, pp. 1826-1835, Aug. 2016.

[58] Y. Cui, R. G Kavasseri, and S. M Brahma, "Dynamic state estimation assisted out-of-step detection for generators using angular difference," IEEE Trans. Power Del., vol. 32, no. 3, pp. 14411449, Jun. 2017.

[59] M. A. M. Ariff, B. C. Pal and A. K. Singh, "Estimating dynamic model parameters for adaptive protection and control in power system," IEEE Trans. Power Syst., vol. 30, no. 2, pp. 829-839, March 2015.

[60] M. A. M. Ariff and B. C. Pal, "Adaptive protection and control in the power system for wide-area blackout prevention," IEEE Trans. Power Del., vol. 31, no. 4, pp. 1815-1825, Aug. 2016.

[61] Y. Liu, A. P. Meliopoulos, R. Fan, L. Sun, Z. Tan, "Dynamic state estimation based protection on series compensated transmission lines", IEEE Trans. Power Del., vol. 32, no. 5, pp 2199-2209, Oct. 2017.

[62] Y. Liu, A. P. Meliopoulos, L. Sun and R. Fan, "Dynamic state estimation based protection on mutually coupled transmission lines," CSEE Journal Power Energy Syst., vol. 2, no. 4, pp. 6-14, Dec. 2016.

[63] R. Fan, Y. Liu, A. P. Meliopoulos, L. Sun, Z. Tan and R. Huang, "Comparison of transformer legacy protective functions and a dynamic state estimation-based approach", Electric Power Syst. Research, 2020.

[64] V. Terzija, G. Preston, V. Stanojević, N. I. Elkalashy, and M. Popov, "Synchronized measurements-based algorithm for short transmission line fault analysis", IEEE Trans Smart Grid, vol. 6, no. 17 , pp. 2639-2648, Nov. 2015.

[65] Y. Liu, A. P. Meliopoulos, Z. Tan, L. Sun and R. Fan, "Dynamic state estimation-based fault locating on transmission lines", IET Gener. Transm. Distrib., vol. 11, no. 17, pp. 4184-4192, Nov. 2017.

[66] R. Fan, Y. Liu, R. Huang, R. Diao and S. Wang, "Precise fault location on transmission lines using ensemble Kalman filter", IEEE Trans. Power Del., vol. 33, no. 6, pp. 3252-3255, Dec. 2018.

[67] B. Wang, Y. Liu, D. Lu, K. Yue and R. Fan, "Transmission line fault location in MMC-HVDC grids based on dynamic state estimation and gradient descent", IEEE Trans. Power Del., 2020.

[68] M. B. Djuric, Z. M. Radojevic, and V. V. Terzija. "Time domain solution of fault distance estimation and arcing faults detection on overhead lines," IEEE Trans. Power Del., vol 14, no. 1, pp. 60 67, Feb. 1999.

[69] H. F. Albinali and A. P. Meliopoulos, "Resilient protection system through centralized substation protection," IEEE Trans.
Power Del., vol. 33, no. 3, pp. 1418-1427, June 2018.

[70] A. P. Deshpande, S. C. Patwardhan, S. S. Narasimhan, "Intelligent state estimation for fault tolerant nonlinear predictive control," Journal of Process Control, vol. 19, pp. 187-204, 2009. 